\title{
THE APPLICATION OF SYNERGISTIC EXTRACTION TO ANALYTICAL CHEMISTRY
}

\author{
H. Akaiwa and H. Kawamoto \\ Department of Chemistry, Faculty of Technology, \\ Gunma University, Kiryu, Gunma 376, Japan
}

\section{CONTENTS}

INTRODUCTION

SYNERGISTIC EXTRACTION EQUILIBRIA

ADVANTAGE OF SYNERGISM

Enhancement of extractability

Widening the optimum extraction $\mathrm{pH}$ range

Extraction with lower concentrations of chelating agent

Stabilization of an extracted species by forming an adduct

Kinetic effect

APPLICATION TO PRACTICAL ANALYSIS

Extractive spectrophotometry

72

Mutual separation of divalent metal ions

Group extraction

80

Miscellaneous

83

CONCLUSION

83

REFERENCES

85 


\section{INTRODUCTION}

A chelate extraction system which is useful for practical analysis should extract a metal ion almost quantitatively by a single equilibration of two phases. However, it is often difficult to achieve the above task by using a single extractant. For example, the extraction of strontium and/or magnesium ions cannot be performed quantitatively by using 8-quinolinol ( $\mathrm{HOx})$ alone, and two different attempts have been made to improve the extraction of these metals. The first was based on the formation of a hydrophobic self-adduct $\operatorname{Sr}(\mathrm{Ox})_{2}(\mathrm{HOx})_{2}$ by employing an excess of the reagent $/ 1 /$. The analytical usage of the self-adduct extraction is, however, limited and, therefore, beyond the scope of this review. The other quantitative extraction of bis(8-quinolinolato)magnesium(II) used the addition of n-butylamine $/ 2 /$. The successful result of the latter example might be due to the cooperative effect of these two extractants. Analytical application of ternary complexes had been made using copper(II)-salicylic acid-pyridine /3/ and iron(II)-dimethylglyoxime-pyridine $/ 4$ / systems, before the term "synergism" was introduced into the field of solvent extraction chemistry $/ 5 /$.

According to the literal meaning of the word, all extraction mechanisms based on the formation of a ternary complex can be classified as synergism; however, the term "synergism" employed in this review is restricted to a phenomenon in which the extractability of a metal chelate is increased by adding a neutral ligand. Even now, the finding of a new extraction system which is important to analytical chemistry depends mainly upon the pioneer's experience, but the wide range of information obtained through physico-chemical studies must be a significant factor for further developments in the analytical application of synergistic extraction. In the present review, emphasis will be laid on the, most common, synergism caused by the formation of a coordinatively saturated adduct. Less common systems including the formation of adducts containing inorganic anions, extraction of chelates in the presence of bulky organic cations, the use of two chelating agents and some other systems have already been reviewed by Hála $/ 6 /$.

\section{SYNERGISTIC EXTRACTION EQUILIBRLA}

Generally speaking, synergism may be expected in those cases where a chelating agent can exactly satisfy the charge of a metal ion to form an uncharged extractable complex but leaving some of the coordination 
sites of the metal for solvent molecules that might then be replaced by the synergist. The mechanism of the resulting synergism is generally explained in terms of the formation of an organophilic adduct.

In the case of no additive present, the extraction reaction of a metal ion $\mathbf{M}^{\mathbf{X}}$ is expressed by

$$
\mathrm{M}^{\mathrm{x}+}+\mathrm{xHR}_{\mathrm{org}} \leftrightharpoons \mathrm{MR}_{\mathrm{x}, \mathrm{org}}+\mathrm{xH}^{+}
$$

where HR denotes a chelating agent and the subscript org means that the species exists in the organic phase. For the simple case that all species except $\mathrm{MR}_{\mathrm{x}}$ and $\mathrm{Mx}^{\mathrm{x}}$ can be neglected, the distribution ratio is given by

$$
\mathrm{D}=\frac{\left[\mathrm{MR}_{\mathrm{x}}\right]_{\mathrm{org}}}{\left[\mathrm{M}^{\mathrm{x}}\right]}
$$

The adduct formation equilibrium in synergistic extraction with a mixture of $\mathrm{HR}$ and an additive $\mathrm{S}$ is expressed by

$$
\mathrm{MR}_{\mathrm{x}, \text { org }}+\overline{\mathrm{y}} \mathrm{S}_{\mathrm{org}} \rightleftharpoons \mathrm{MR}_{\mathrm{x}} \mathrm{S}_{\mathrm{y}, \mathrm{org}}
$$

where $\mathrm{MR}_{\mathrm{x}} \mathrm{S}_{\bar{y}}$ represents the average composition of the synergistic complexes in the organic phase and $\bar{y}=\sum_{y=1} y\left[M R_{x} S_{y}\right]_{\text {org }} / y=\sum_{1}$. $\left[M R_{x} S_{y}\right]_{\text {org }}$ is the average number of $S$ bound by $M R_{x}$. A conditional constant for the reaction ( 3 ) is defined by

$$
\beta_{\bar{y}}=\frac{\left[M R_{x} S_{y}^{-}\right]_{o r g}}{\left[M R_{x}\right]_{o r g}[S]_{o r g}^{\bar{y}}}
$$

and the distribution ratio of the above system is given by

$$
D^{*}=\frac{\left[M R_{x}\right]_{\text {org }}+\left[M R_{x} S_{y}^{-}\right]_{\text {org }}}{\left[M^{x^{+}}\right]}
$$

Combining equations (2), (3) and (4), one obtains:

$$
\log \frac{\nu^{k}}{D}=\log \left(1+\beta_{\bar{y}}[S]_{\mathrm{org}}^{\bar{y}}\right)
$$

where $\log D^{*} / D$ is a measure of positive synergism and called the synergistic coefficient (S.C. $\equiv \log D^{*} / D$ ). 
Since adduct formation is an acid-base reaction, the basicity of a ligand added to a Lewis acid metal chelate is the most important factor controlling the stability of the resulting adduct. Fig. 1 shows the relationship between the S.C. values and the basicities of additives for the system nickel-2-thenoyltrifluoracetone (TTA=Htta)-pyridine base $17 \%$. The S.C. values of the pyridine bases agree well with their basicities

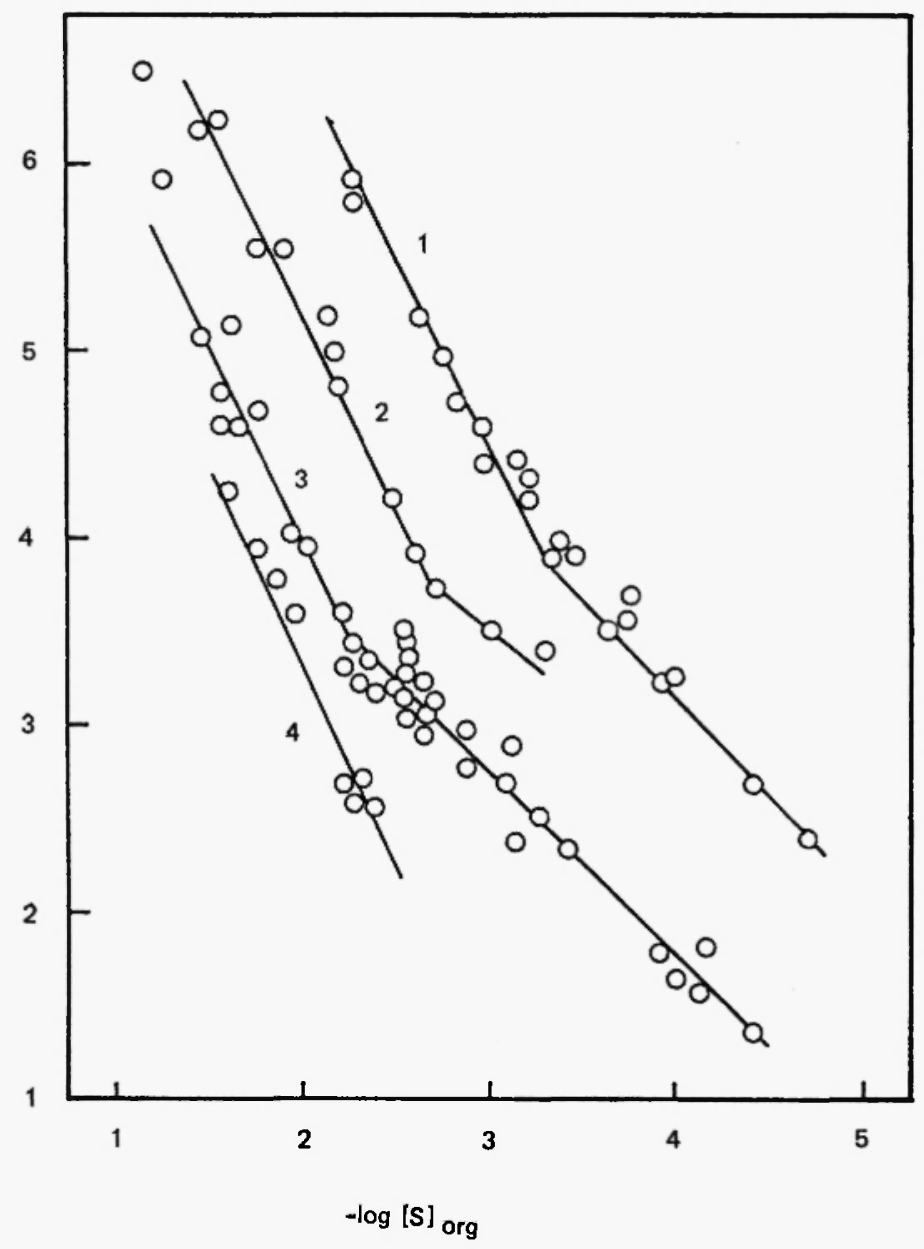

Fig. 1. Synergistic coefficients (S. C.) vs. $-\log [S]_{\text {org. }} 1: \gamma$-picoline, 2: $\beta$-picoline, 3: pyridine, 4: $\alpha$-picoline. 
except for $\alpha$-picoline where the ortho-methyl group sterically hinders the metal chelate from forming an adduct. Similar tendencies were observed for the extraction systems TTA-manganese(II) $/ 8 /$, -iron(II) $/ 9 /$, and -cobalt(II) /10/ -pyridine bases. For additives such as organophosphorus compounds, the situation is quite similar $/ 11 /$. Besides the basicity of the additive, the stability of the synergistic adduct is further influenced by the Lewis acidity of the host chelate and the nature of the diluent $/ 12 /$.

\section{ADVANTAGE OF SYNERGISM}

The addition of a synergist often enhances the extractabilities of coexisting metal ions as well as that of the analyte. This feature of synergistic extraction generally renders its application to practical analysis somewhat difficult. If this problem could be overcome, the inherent nature of synergism would play a valuable role in trace analysis.

\section{Enhancement of extractability}

Upon the addition of a neutral base to a coordination unsaturated metal chelate, the organophilic property of the resulting adduct generally increases. This phenomenon often turns a chelate system of low extractability into a sensitive method of determination. The spectrophotometric determination of manganese(II) with TTA was established by employing a synergistic extraction technique /13/, and, indeed, TTA could not be an important reagent in practical analysis without the aid of synergism.

\section{Widening the optimum extraction $\mathrm{pH}$ range}

As chelate formation is a competing reaction between a metal ion and a proton of the chelating agent an alkaline medium is generally favourable for the formation of the metal chelate. However, metal ions tend to form stable hydroxo-complexes in an alkaline medium and thus interfere with chelate formation. By adding a neutral base to a chelate extraction system, the extraction curve can be shifted to the acidic region. This phenomenon is very useful for practical analysis as illustrated by the manganese(II)-dithizone-1,10-phenanthroline system /14/ (Fig. 2). 


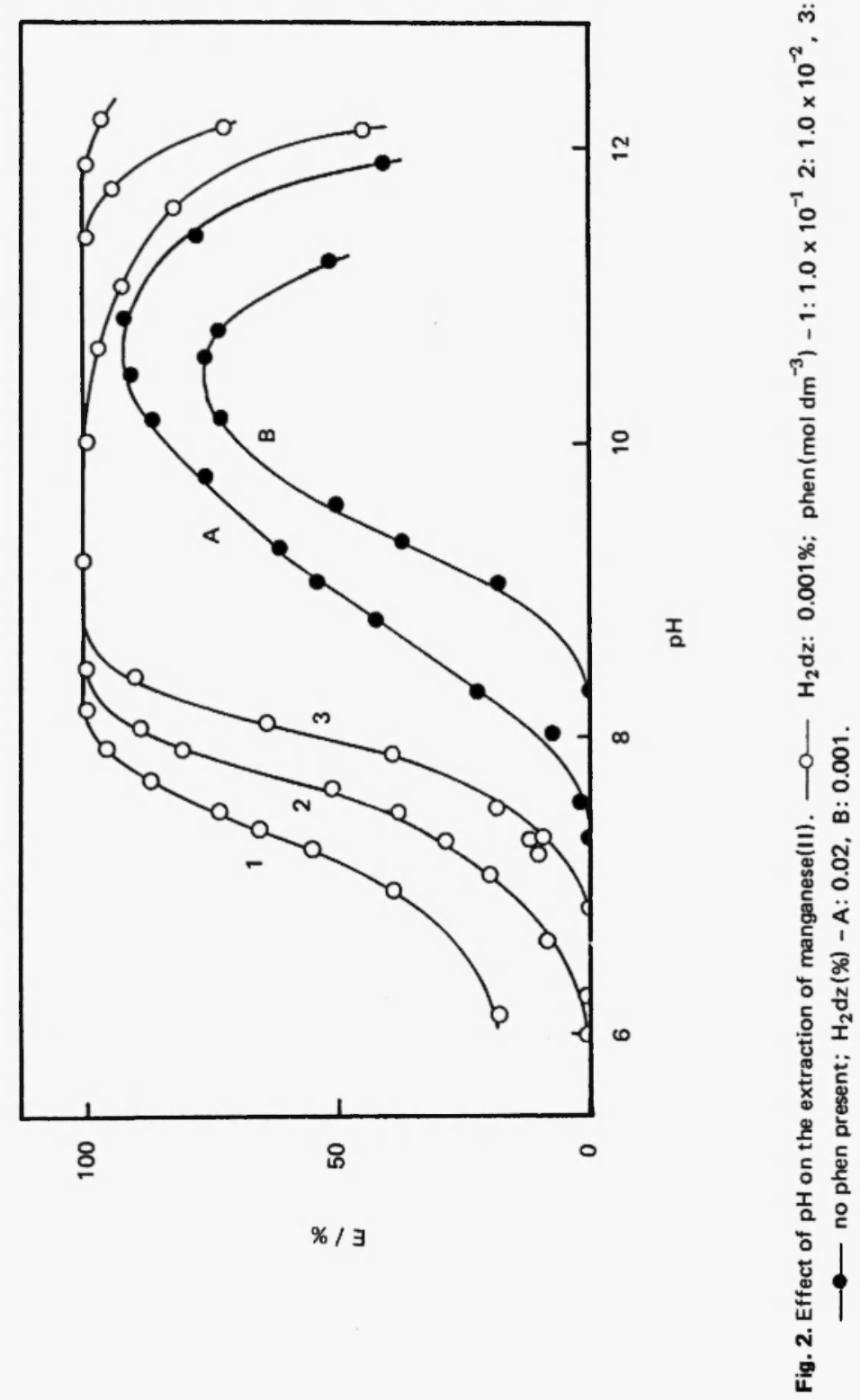




\section{Extraction with lower concentrations of chelating agent}

The same extractability as in the case of a single extractant, but with lower concentration of a chelating agent can be obtained by employing a synergistic extraction system. The use of low concentration of extractant minimizes the reagent blank and thus enhances the sensitivity of spectrophotometry, as reported in the study on the system nickel-TTAisoquinoline /15/. This advantage of synergism was also applied to spectrofluorimetry, e.g. the europium(III) and samarium(III)-naphthoyltrifluoroacetone trioctylphosphine oxide (TOPO) systems /16/.

\section{Stabilization of an extracted species by forming an adduct}

This effect is often useful for increasing the sensitivity of absorbance measurements. In the case in which a residual extractant interferes with the absorbance measurement at an optimum wavelength, the residual reagent can be decomposed by washing the organic phase with an alkaline solution of appropriate concentration. This treatment may not be effective in the absence of synergism since an alkaline solution may decompose not only the residual extractant, but the chelate itself.

In some cases, the use of a synergist stabilizes the oxidation state of a metal ion in an extracted species. For example, in its dithizone extract, cobalt(II).is readily oxidized to cobalt(III), whereas the addition of 1,10-phenanthroline as a synergist prevents such oxidation $/ 17 /$. Stabilization of an oxidation state has also been observed in iron(II)TTA-pyridine base systems $/ 9 /$, which are applied to selective analysis of iron(II) $/ 18 /$.

An increased thermal stability in the rare earth(III)-hexafluoroacetylacetone-tributylphosphate (TBP) system has been utilized in the gas chromatographic separation of rare earth elements $/ 19 /$.

\section{Kinetic effect}

The addition of a neutral ligand to a chelate extraction system sometimes accelerates the extraction. Nickel in a weakly acidic solution cannot be extracted quantitatively into a carbon tetrachloride solution of dithizone even by shaking the mixture for over an hour. But, the dithizone extraction of nickel becomes rapid in the presence of pyridine 120/. A similar effect of $2,2^{\prime}$-dipyridyl was observed in dithizone extractions of zinc $/ 21 /$. 
The application of the kinetic effect of synergism to the mutual separation of divalent metals will be discussed later.

\section{APPLICATION TO PRACTICAL ANALYSIS}

The application of synergistic extraction to practical analysis can be divided into two main categories. One is the improvement of sensitivity in extractive spectrophotometry, and the other, the mutual separation of trace elements.

\section{Extractive spectrophotometry}

As mentioned previously, cobalt(II) cannot normally be extracted quantitatively with TTA due to the hydrophilic property of the chelate formed. However, quantitative extraction of cobalt(II) can be attained by the addition of acetone, and this system is utilized to establish a method for the spectrophotometric determination of cobalt (II) with TTA /22/. Unfortunately, in this case, a comparatively large amount of TTA is needed and this interferes with the absorbance measurement of the analytical species. The problem can be overcome by using pyridine instead of acetone, that is to say, cobalt(II) is extracted quantitatively even with a low concentration of TTA. As a result, the interference caused by the reagent blank in the absorbance measurement of the synergistic adduct $\mathrm{Co}(\mathrm{tta})_{2}(\mathrm{py})_{2}$ in benzene is eliminated to a large extent $/ 23 /$. Moreover, stripping the organic phase with sodium hydroxide makes it possible to transfer the residual TTA in to the aqueous phase, whereas the adduct remains quantitatively in the organic phase. As a consequence, absorbance measurement at the maximum wavelength of the adduct becomes feasible and a remarkable improvement in the sensitivity results $/ 24 /$. Table 1 summarizes the sensitivities of some methods with TTA. It is clear that the sensitivities of methods using synergistic extraction are consistently high especially in those cases in which the organic phase was treated with an alkaline solution. For example, in the case of copper, the increase in the sensitivity is more than 100 fold.

Sometimes synergism plays an important role in the extraction of metal dithizonates. Although dithizonatomanganese(II) has not been used for analytical purposes, the extractability of manganese(II) is enhanced remarkably by employing the synergistic effect of added 1,10phenanthroline and quantitative extraction is then achieved over a wide 
TABLE 1. Improvement in sensitivities of some spectrophotometric methods obtained by synergistic extraction

\begin{tabular}{|c|c|c|c|c|c|}
\hline Metal ion & Diluent & Additive & $\begin{array}{c}\text { Molar } \\
\text { absorptivity }\end{array}$ & $\begin{array}{l}\text { Measured } \\
\text { waveleng th(nm) }\end{array}$ & Reference \\
\hline Cobalt (II) & $\begin{array}{l}\text { Benzene } \\
+ \text { acetone }\end{array}$ & None & $8.0 \times 10^{2}$ & 430 & 22 \\
\hline$"$ & Benzene & Pyridine & $4.2 \times 10^{3}$ & 390 & 23 \\
\hline$" *$ & Benzene & Pyridine & $3.0 \times 10^{4}$ & 340 & 24 \\
\hline Nickel & $\begin{array}{l}\text { Benzene } \\
+ \text { acetone }\end{array}$ & None & $6.2 \times 10^{2}$ & 420 & 43 \\
\hline$"$ & Benzene & Isoquinoline & $2.3 \times 10^{3}$ & 390 & 15 \\
\hline$" *$ & $\begin{array}{l}\text { Cyclo- } \\
\text { hexane }\end{array}$ & Piperidine & $2.4 \times 10^{4}$ & 350 & 44 \\
\hline Copper (II) & Benzene & None & $2.2 \times 10^{2}$ & 430 & 45 \\
\hline$" *$ & $\begin{array}{l}\text { Cyclo- } \\
\text { hexane }\end{array}$ & Piperidine & $2.8 \times 10^{4}$ & 340 & 46 \\
\hline
\end{tabular}

HR=TTA; * The organic phase was stripped with $\mathrm{NaOH}$.

range of $\mathrm{pH}$, as can be seen in Fig. 2. The resulting adduct $\mathrm{Mn}(\mathrm{Hdz})_{2}$ (phen) is stable enough for the spectrophotometric determination of manganese(II) $/ 14 /$. The excellent method of spectrophotometric determination of nickel with dithizone is based on the addition of 1,10 phenanthroline, which accelerates the extraction of nickel and at the same time causes a favourable change in the spectrum of the host chelate $/ 25 /$. Synergism in the extraction of dithizonates has also been found in the cases of vanadium(IV,V) $/ 27 /$, iron(II) $/ 27 /$, cobalt(II) $/ 17 /$, cadmium $/ 28 /$ and lead $/ 29 /$.

One of the disadvantages of synergistic extraction is that the addition of a synergist usually decreases the selectivity for a particular metal ion in the extractive separation. On the other hand, it can be said that the addition of a synergist to a chelate extraction system may increase the number of factors controlling the selectivity. This will be discussed in the next section.

\section{Mutual separation of divalent metal ions}

Equations (7) and (8) show the extraction reaction of two divalent 
metal ions, $\mathrm{M}^{2+}$ and $\mathrm{N}^{2+}$ that should be separated from each other. The extraction constant for $\mathrm{MR}_{2}$ and the stability

$$
\begin{aligned}
& \mathrm{M}^{2+}+2 \mathrm{HR}_{\mathrm{org}}+\mathrm{mS}_{\mathrm{org}} \stackrel{\mathrm{K}_{\mathrm{Ex,m}}^{\mathrm{M}}}{\rightleftharpoons} \mathrm{MR}_{2} \mathrm{~S}_{\mathrm{m}, \mathrm{org}} \\
& \mathrm{N}^{2+}+2 \mathrm{HR}_{\mathrm{org}}+\mathrm{nS}_{\mathrm{org}} \stackrel{\mathrm{K}_{\mathrm{ex,n}, \mathrm{n}}^{\mathrm{N}}}{\rightleftharpoons} \mathrm{NR}_{2} \mathrm{~S}_{\mathrm{n}, \mathrm{org}}
\end{aligned}
$$

constant for $\mathrm{MR}_{2} S_{m}$ in the organic phase are expressed by $\underset{\mathbf{K}}{-\mathrm{Mx}}$ and $\beta_{\mathrm{m}}^{\mathrm{M}}$, respectively, from which the equation (9) can be obtained.

$$
K_{e x, m}^{M}=K_{e x}^{M} \cdot \beta_{m}^{\mathrm{M}}
$$

In most cases in synergistic extraction analysis, a high concentration of the synergist is employed and the concentration of the chelating agent is kept as low as possible. Therefore, in the ideal case, the distribution atio may be expressed by equation (10). Meanwhile, by using the distribution ratio $\mathrm{D}^{\mathrm{N}}$ for the metal

$$
\mathrm{D}^{\mathrm{M}}=\frac{\left[\mathrm{MR}_{2} \mathrm{~S}_{\mathrm{m}}\right]_{\text {org }}}{\left[\mathrm{M}^{2+}\right]}
$$

ion $\mathrm{N}^{2+}$, the separation factor $\mathrm{S}$ can be represented by equation (11).

$$
S=\frac{D^{M}}{D^{N}}
$$

Taking logarithms we obtain

$$
\log S=\log \frac{K_{e x}^{M}}{K_{e x}^{N}}+\log \frac{\beta_{\frac{m}{M}}^{M}}{\beta_{n}^{N}}+(m-n) \log [S]_{0 r \tilde{z}}
$$

Where $\log \mathrm{S}$ is a measure of the selectivity for the two metal ions; if this value exceeds 4 , metal ion $\mathrm{M}^{2+}$ can be separated quantitatively from $\mathrm{N}^{2+}$.

The first term in the right hand side of the equation (12) corresponds to the selectivity in the absence of a synergist. Consequently, for a particular chelating agent, the selectivity in a synergistic extraction is controlled by the stability constants $\beta$ of the adducts and the concentration of the synergist. The last term is important only when $m \neq n$. For example, the extraction system which involves zinc and cadmium dithizonates is a case in point in which zinc does not form an adduct but 
cadmium does. Yet, the most important term controlling the selectivity of the synergistic extraction is the second one, which is affected by five main factors; viz. (1) the nature of the diluent used in the chelate extraction system, (2) the basicity of the synergist, (3) the steric hindrance caused by an additive in adduct formation, (4) the Lewis acid nature of the host chelate, and (5) the kinetic effect. These factors are now discussed in some detail.

The nature of the diluent: The effect of a diluent in a chelate extraction system is sometimes successfully explained in terms of the theory of regular solutions $/ 30 /$. However, at present, the most suitable diluent for a given extraction system must be chosen empirically for practical purposes. In general, the resulting adduct is the most stable in cyclohexane and least stable in chloroform. Therefore, in the case of a synergistic extraction of a single metal ion, cyclohexane is considered to be the best diluent, but for separation purposes this is not necessarily the case.

The effect of the diluent in the monothiothenoyltrifluoroacetone (STTA) extraction in the absence of synergism is shown in Table 2.

TABLE 2. Effect of diluents (I)

\begin{tabular}{lccc}
\hline Diluent & Cd $\log \mathrm{K}_{\mathrm{ex}} \mathrm{Zn}$ & $\log \left(\mathrm{D}_{\mathrm{zn}} / \mathrm{D}_{\mathrm{Cd}}\right)$ \\
\hline $\mathrm{CCl}_{4}$ & -6.00 & -4.30 & 1.70 \\
$\mathrm{C}_{2} \mathrm{HCl}_{3}$ & -5.78 & -4.28 & 1.50 \\
$\mathrm{C}_{6} \mathrm{H}_{5} \mathrm{Cl}$ & -5.52 & -4.16 & 1.36 \\
$\mathrm{C}_{6} \mathrm{H}_{5}\left(\mathrm{CH}_{3}\right)$ & -5.46 & -4.26 & 1.20 \\
$\mathrm{CHCl}_{3}$ & -5.40 & -4.20 & 1.20 \\
$\mathrm{CH}_{2} \mathrm{ClCH}_{2} \mathrm{Cl}$ & -5.24 & -4.40 & 0.84 \\
$\mathrm{CH}_{2} \mathrm{Cl}_{2}$ & -4.96 & -4.20 & 0.76 \\
$\mathrm{C}_{2} \mathrm{H}_{5} \mathrm{Br}$ & -4.80 & -4.16 & 0.64 \\
$\mathrm{C}_{2} \mathrm{H}_{2} \mathrm{Br}$ & -4.70 & -4.06 & 0.64 \\
\hline
\end{tabular}

HR $=\operatorname{STTA}\left(5.0 \times 10^{-3} \mathrm{~mol} \mathrm{dm}^{-3}\right) ;$ no additive present

The extraction constant, $\mathrm{K}_{\mathrm{ex}}$ for zinc remains almost unchanged regardless of the kind of diluent. On the other hand, $\mathrm{K}_{\mathrm{ex}}$ for cadmium ranges from -6.0 to -4.7 . A comparison of the values of the last column shows that the highest selectivity is obtained by using carbon tetra- 
chloride as a diluent. It should be noted that the distribution ratio for zinc is consistently larger than that of cadmium. Table 3 lists the effect of the diluent on the synergistic extraction of zinc and cadmium in the STTA-TOPO system. The half extraction $\mathrm{pH}$ for cadmium is

TABLE 3. Effect of diluents (II)

\begin{tabular}{lcc}
\hline Diluent & $\mathrm{pH}_{1 / 2}(\mathrm{Cd})$ & $\log \left(\mathrm{D}_{\mathrm{Cd}} / \mathrm{D}_{\mathrm{Zn}_{n}}\right)$ \\
\hline $\mathrm{n}-\mathrm{C}_{5} \mathrm{H}_{12}$ & 1.60 & 1.46 \\
$\mathrm{C}_{6} \mathrm{H}_{12}$ & 1.48 & 1.36 \\
$\mathrm{n}-\mathrm{C}_{6} \mathrm{H}_{14}$ & 1.47 & 1.32 \\
$\mathrm{C}_{6} \mathrm{H}_{3}\left(\mathrm{CH}_{3}\right)_{3}$ & 2.21 & 0.58 \\
$\mathrm{CCl}_{2}=\mathrm{CCl}_{2}$ & 2.16 & 0.56 \\
$\mathrm{C}_{6} \mathrm{H}_{6}$ & 2.38 & 0.46 \\
$\mathrm{CCl}_{4}$ & 2.45 & 0.46 \\
$\mathrm{C}_{6} \mathrm{H}_{5}\left(\mathrm{CH}_{3}\right)$ & 2.30 & 0.44 \\
$\mathrm{C}_{6} \mathrm{H}_{5} \mathrm{Cl}$ & 2.44 & 0.18 \\
$\mathrm{CHCl}_{3}$ & 3.63 & 0.18 \\
$\mathrm{CH}_{2} \mathrm{Cl}_{2}$ & 3.07 & 0.18 \\
$\mathrm{CH}_{2} \mathrm{ClCH}_{2} \mathrm{Cl}$ & 2.96 & 0.12 \\
\hline
\end{tabular}

HR=STTA $\left(5.0 \times 10^{-3} \mathrm{~mol} \mathrm{dm}^{-3}\right) ; S=T O P O\left(1.0 \times 10^{-2} \mathrm{~mol} \mathrm{dm}^{-3}\right)$

noticeably lower for each diluent than is the case of extraction without synergism. For example, the half extraction $\mathrm{pH}$ of cadmium-STTA in carbon tetrachloride is 5.3 , and that of synergistic extraction is 2.45 . This means that cadmium can be extracted without any troubles such as the hydrolysis of coexisting ions by the aid of synergism. In addition, it is obvious from the separation factor values that cadmium is more easily extracted than zinc in the case of synergistic extraction. The high selectivity obtained in n-pentane or cyclohexane is related to the high stability of the resulting adduct in these diluents. However, in the case of the cobalt (II) and nickel-TTA-pyridine base systems, the situation is different $/ 31 /$. The above two examples show the difficulty that exists in choosing a proper diluent for selective separation.

The basicity of the synergist: The separation factor obtained in the zinc and cadmium-STTA system is plotted against the acid dissociation exponent ( $\mathrm{pKa}$ ) of the synergist, and the results are given in Fig. $\therefore$. This 


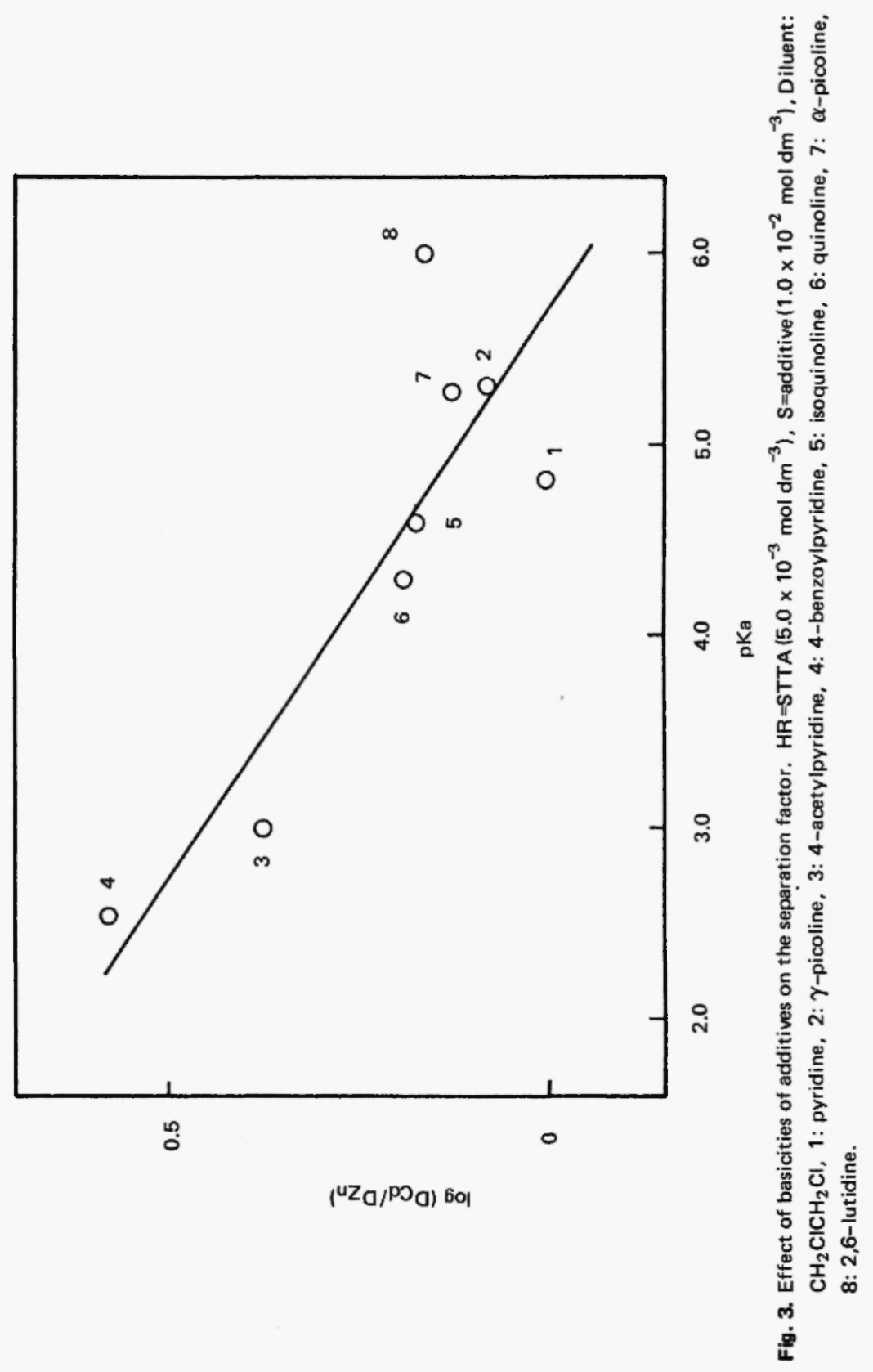


clearly shows that the lower the basicity of the synergist, the higher the separation factor, i.e., higher selectivity is obtained by employing a less basic synergist. The separation factor obtained by using TOPO is almost the same as that with isoquinoline. Therefore, the use of a highly basic synergist such as piperidine does not necessarily provide high selectivity.

Under the conditions employed in Fig. 3, the third term in the right hand side of equation (12) may contribute to the selectivity, particularly in a low pKa region.

The structure of the synergist: By comparing two sets of points in Fig. 3, e.g. isoquinoline with quinoline, and $\gamma$-picoline with $\alpha$-picoline, one may find that the bulkier substituent does not make a significant contribution to the selectivity in STTA extraction. However, the result of TTA extraction shows that the steric factor plays a somewhat important role in the separation. The degree of steric hindrance caused by the ortho-substituent using half extraction $\mathrm{pH}$ values is indicated in Table 4. The $\Delta \mathrm{pH}_{1 / 2}^{*}$ values were obtained against the half extraction $\mathrm{pH}$ of

TABLE 4. Steric hindrance caused by an or tho-substituent

\begin{tabular}{llll}
\hline Additive & \multicolumn{2}{c}{$\Delta \mathrm{pH}_{1 / 2^{*}} \mathrm{Cd}$} & $\Delta \mathrm{pH}_{1 / 2^{* *}}$ \\
\hline py & 0.0 & 0.0 & 0.60 \\
a-picoline & 0.36 & 0.34 & 0.58 \\
2-ethylpy & 0.28 & 0.64 & 0.96 \\
2-benzylpy & 0.60 & 1.02 & 1.02 \\
2,6-lutidine & 0.70 & 0.90 & 0.80 \\
\hline
\end{tabular}

HR=TTA; Diluent= $\mathrm{C}_{6} \mathrm{H}_{6} ;{ }^{*} \mathrm{pH}_{1 / 2}(\mathrm{~S})-\mathrm{pH}_{1 / 2}$ (py);

** $\mathrm{pH}_{1 / 2}(\mathrm{Cd})-\mathrm{pH}_{1 / 2}(\mathrm{Zn})$

the TTA-pyridine system as standard. Those values are considered to be a quantitative measure of the degree of steric hindrance caused by the orthosubstituent. A large value of the $\Delta \mathrm{pH}_{1 / 2}^{*}$ corresponds to significant steric hindrance. For example, in the case of cadmium, the increasing order of the steric hindrance is methyl, ethyl, benzyl and so on. This order corresponds to that of the bulk of the substituent.

The nature of the host metal chelate: The stability constants of some adducts in carbon tetrachloride are listed in Table 5. In the case 
of TTA which has o,o-donor atoms, zinc forms more stable adducts than does cadmium. Consequently even in a synergistic extraction system, zinc is extracted preferentially to cadmium. On the other hand, in

TABLE 5. Stability constants for some adducts

\begin{tabular}{lccc}
\hline Adduct & Diluent & $\log \beta$ & Reference \\
\hline Zn(TTA $)_{2}$ TBP & $\mathrm{CCl}_{4}$ & 4.34 & 47 \\
Cd(TTA $)_{2} \cdot$ TOPO & $\mathrm{CCl}_{4}$ & 1.02 & 47 \\
Zn(STTA $)_{2} \cdot$ TOPO & $\mathrm{CCl}_{4}$ & 5.64 & 29 \\
Cd(STTA $)_{2} \cdot$ TOPO & $\mathrm{CCl}_{4}$ & 7.70 & 29 \\
\hline
\end{tabular}

the STTA case the stability constant for cadmium is 100 times larger than that for zinc. These facts suggest that the selection of a proper chelating agent is also one of the most important factors affecting selectivity in synergistic extraction.

As can be seen in Table 6, the mutual separation of zinc and cadmium was not successful without adding the synergist. In contrast, in

TABLE 6. Effect of a chelating agent on the separation

\begin{tabular}{lll}
\hline Chelating agent & Additive & $\begin{array}{c}\text { Metal ion extracted } \\
\text { preferentially }\end{array}$ \\
\hline TTA & None & $\mathrm{Zn}$ \\
$"$ & $2,6-l u t^{*}$ & $\mathrm{Zn}$ \\
STTA & None & $\mathrm{Zn}$ \\
Dithizone & dipy* & $\mathrm{Cd}$ \\
$"$ & None & $\mathrm{Zn}$ \\
\hline
\end{tabular}

* Quantitative separation was achieved.

the synergistic extraction systems of STTA and dithizone, cadmium could be extracted preferentially to zinc and quantitative separation became possible in both cases. At this point, the reason for the above facts cannot be explained because of our lack of the knowledge of the structures of the host and adduct, or the change in hydration number during the extraction processes. 
Practical examples of mutual separation: Dithizone has been widely used for the colorimetric determination of both zinc and cadmium and the extraction behaviour of these metal ions closely resembles each other. Up to now, the mutual separation of zinc and cadmium has been based on zinc not reacting with dithizone in alkaline medium because of the formation of the zincate ion. 2,2'-dipyridyl (dpy) reacts with dithizonatocadmium to form a $1: 1$ adduct $\mathrm{Cd}(\mathrm{Hdz})_{2} \mathrm{dpy}$, but in the extraction of dithizonatozinc, almost no synergism is observed. In the ideal case, cadmium is transferred to the organic phase quantitatively as a synergistic adduct, whereas zinc remains in the aqueous phase as a complex cation with $2,2^{\prime}$-dipyridyl. The above condition is realized when the $2,2^{\prime}$-dipyridyl concentration equals $0.1 \mathrm{~mol} \mathrm{dm}^{-3}$, and the difference in the half extraction $\mathrm{pH}$ for zinc and cadmium reaches 2.0. At $\mathrm{pH} 5.0$, quantitative separation of cadmium from zinc with a mixture of dithizone and $2,2^{\prime}$-dipyridyl has been achieved $/ 21$ / in this way.

On the basis of the successful use of $2,2^{\prime}$-dipyridyl, an attempt was made to examine 1,10-phenanthroline similarly. Fig. 4 shows the extraction curves of zinc and cadmium obtained using a mixture of dithizone and 1,10-phenanthroline. Comparison of these curves shows that the optimum $\mathrm{pH}$ range for the separation is wider in this case. This means that 1,10-phenanthroline is a better synergist for this separation $132 /$. However, the possibility of extracting cadmium as an ion-pair, especially in a solvent which has a relatively high dielectric constant, e.g. chloroform $/ 28 /$, should also be borne in mind.

Kinetic factors: In general, the extraction constant of a cobalt(II) chelate is of the same order of magnitude as that of the same nickel chelate, and a similar situation is also observed in synergistic adducts of these metals. The unsuccessful mutual separation of these metal ions with a mixture of TTA and pyridine is probably due to this reason $/ 33 /$. However, complex formation with cobalt (II) proceeds 100 times faster than with nickel(II), and almost the same difference in reaction rate is observed in synergistic extraction/34/. Fig. 5 shows that cobalt(II) can be separated from nickel by controlling the extraction period $/ 35 /$. This is an example in which a kinetic factor controls the selectivity of a synergistic extraction.

\section{Group extraction}

The simultaneous extraction of several metal ions can also be performed with the aid of synergism. In the atomic absorption spectro- 


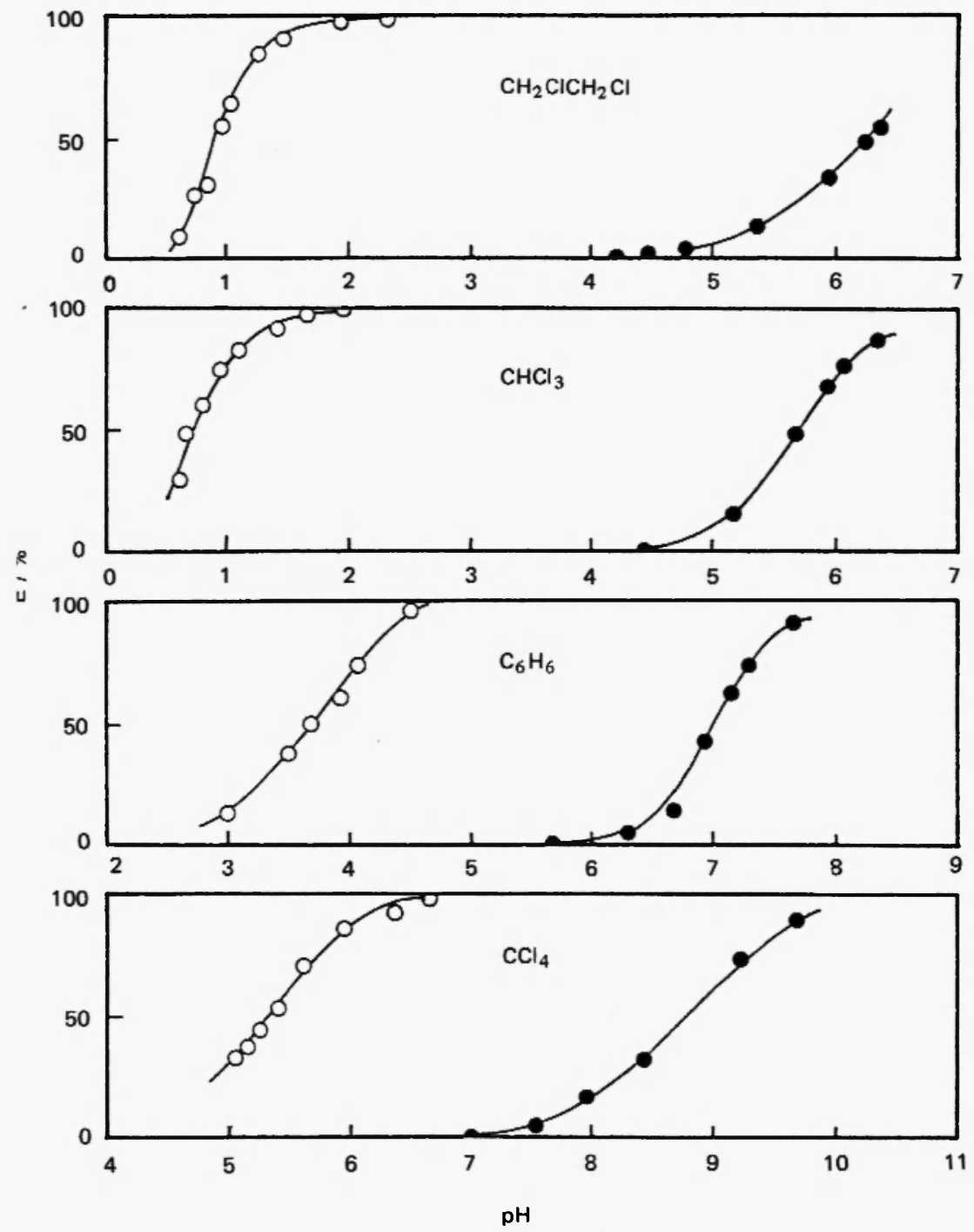

Fig. 4. Extraction curves. $\mathrm{H}_{2} \mathrm{dz}: 0.002 \%$, phen: $0.1 \mathrm{~mol} \mathrm{dm} \mathrm{m}^{-3},-\mathrm{O}-\mathrm{Cd}$, $\longrightarrow$ : Zn. 


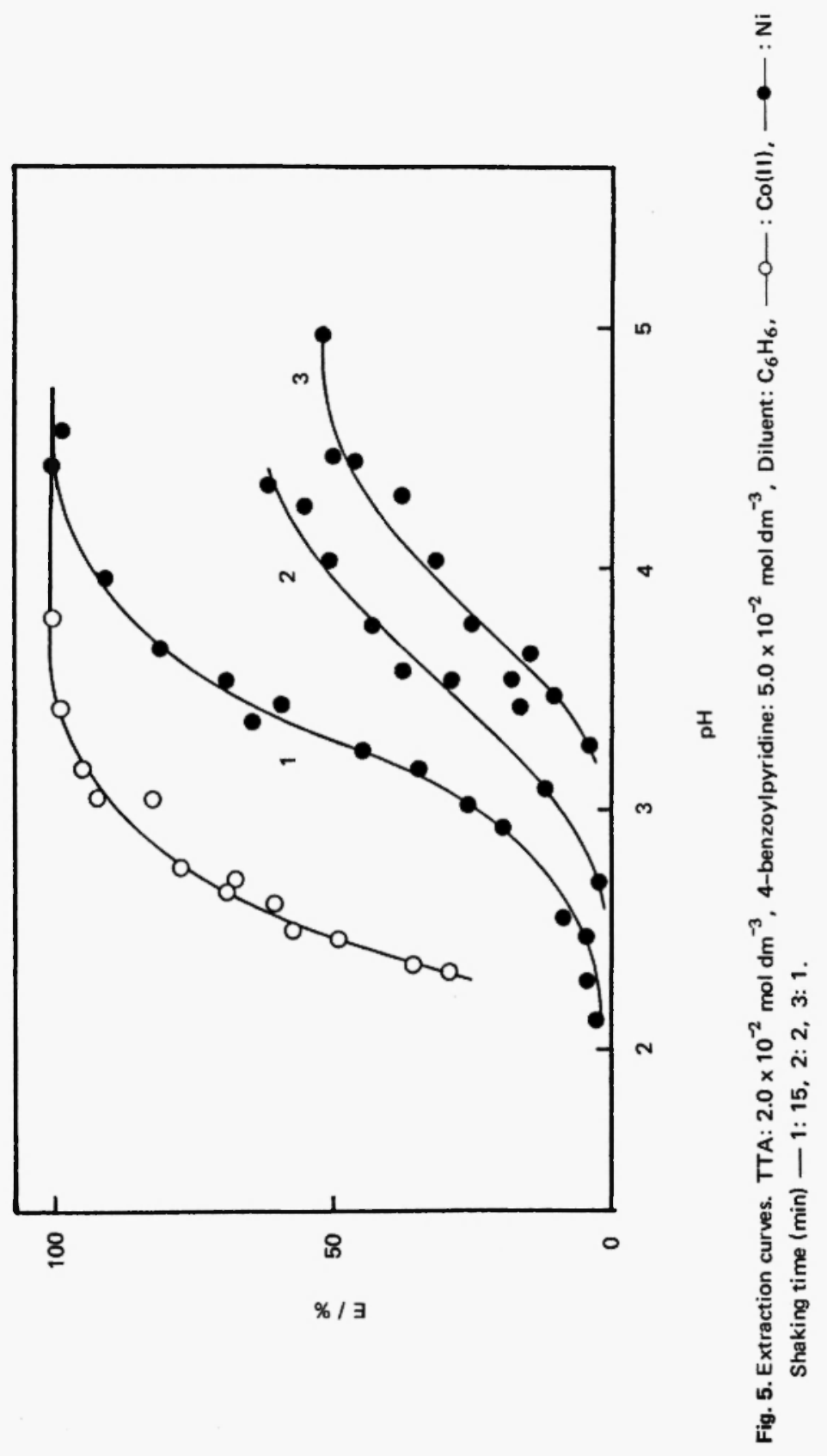


photometric (AAS) determination of calcium in a steel sample, interfering ions such as iron(III), chromium(III) and nickel can be removed effectively by extracting them with acetylacetone and pyridine $/ 36 /$. Although group extraction of selected metal ions prior to flame spectroscopic analysis is also reported $/ 37 /$, the application of synergistic extraction to flame spectroscopy is practically limited, so far a solvent having an oxygen atom is employed as the diluent $/ 38 /$.

In relation to group extraction, synergism is also effective in the preconcentration of trace metal ions before instrumental analysis. The concentration factor of trace amounts of cadmium in natural water samples was increased by a factor of $10^{4}$ using the dithizone-TOPO system /39/:

\section{Miscellaneous}

Adduct formation sometimes changes the absorption spectrum of the host chelate, and as a result the extraction system may become useful for analytical purposes. The stable charge-transfer spectrum of the $\mathrm{Fe}(\mathrm{tta})_{2}(\mathrm{py})_{2} / 9 /$ and the ligand spectrum of the iron(II)-dibenzoylmethane-TOPO system /40/ have both been successfully used for the determination of iron in water. The brown colour of the primary dithizonatonickel $\mathrm{Ni}(\mathrm{Hdz})_{2}$ can be converted to red by the addition of pyridine, and the resulting complex is stable enough to be used for the determination of nickel $/ 20 \%$. In the same system, the use of 1,10 . phenanthroline is more suitable for the analysis $/ 25 /$. Moreover, it was found that $\mathrm{Ni}(\mathrm{Hdz})_{2}$ which is kinetically inert toward back-extraction with mineral acid becomes labile in the presence of 1,10-phenanthroline. This behaviour can be utilized to separate nickel from cobalt(II) and other heavy metal ions $/ 41 /$. Even hexafluoroacetylacetone which has a poor complexing power, has been applied to a substoichiometric isotope dilution method in which copper(II), zinc and europium was extracted by the aid of the synergistic effect of TOPO /42/. Further development of substoichiometry using synergism is promising. In some cases a new analytical reaction can be found by an appropriate combination of two extractants, e.g. the vanadium (IV,V)-dithizone-TOPO 126/ and iron(II)-dithizone-1,10-phenanthroline systems /27/.

\section{CONCLUSION}

In this brief review, the authors have tried to show that synergism in 
chelate extraction is useful not only for spectrophotometry, but for mutual separation of some metal ions.

It is often difficult to find out an ideal chelating agent for a given analytical purpose. In such cases, making use of a masking agent is the only attempt that has been made, so far, to improve the selectivity of methods of high sensitivity. From the above discussion, however, it can be seen that it may be equally or even more use ful to use a synergist in the extraction system. A careful survey of the factors influencing selectivity in synergistic extraction will sometimes make it possible to reach a successful goal. 


\section{REFERENCES}

1. DYRSSEN, D. Svensk. Kem. Tidskr. 67, 311 (1955).

2. UMLAND, F. and HOFFMAN, W. Anal. Chim. Acta., 17, 234 (1957).

3. GORDIEYEFF, V. A. Anal Chem., 22, 1166 (1950).

4. OHI, S. Nippon Kagaku Kaishi, 75, 1069 1954).

5. BLAKE, C.A., BAES, C.F., BROWN, K.B., COLEMAN, C.F. and WHITE, J. Proceedings of the Second International Conference on the Peaceful Uses of Atomic Energy, Geneva, 28, 289 (1958).

6. HALA, J.J. Radioanal. Chem., 51, 15 (1979).

7. KAWAMOTO, H. and AKAIWA, H.J. Inorg. Nucl. Chem., 31, 1141 (1969).

8. KAWAMOTO, H. and AKAIWA, H.J. Inorg. Nucl. Chem., 30, 3065 (1968).

9. KAWAMOTO, H. and AKAIWA, H. Nippon Kagaku Kaishi, 80, 585 (1968).

10. IR VING, H.M.N.H. Solvent Extraction Chemistry, North-Holland Publishing, Amsterdam, p. 91 (1967).

11. KE, C.H. and LI, N.C.J. Inorg. Nucl. Chem., 28, 2255 (1966).

12. HEALY, T.V.J. Inorg. Nucl. Chem. 19, 328 (1961).

13. AKAIWA, H. and KAWAMOTO, H. Bunseki Kagaku, 16, 359 (1967).

14. AKAIWA, H., KAWAMOTO, H. and KOGURE, S. Bunseki Kagaku, 28, 498 (1979).

15. AKAIWA, H., KAWAMOTO, H. and HARA, M. Bunseki Kagaku, 17, 183 (1968).

16. ShIGeMATSU, T., MATSUI, M. and WAKE, R. Anal. Chim. Acta, 46, 101 (1969).

17. AKAIWA, H., KAWAMOTO, H. and HIYAMUTA, E. Nippon Kagaku Kaishi $1979,1716$.

18. AKAIWA, H., KAWAMOTO, H. and HARA, M. Anal. Chim. Acta, 43, 297 (1968).

19. BUTTS, W.C. and BANKS, C.V. Anal. Chem., 42, 133 (1970).

20. AKAIWA, H., KAWAMOTO, H. and HARA, M. Nippon Kagaku Kaishi, 90, 186 (1969).

21. AKAIWA, H., KAWAMOTO, H., OGURA, K. and KONISHI, M. Bunseki Kagaku, 27, 329 (1978).

22. DE, A.K. and MAJUNDAR, S.K. Anal. Chim. Acta, 27, 153 (1962).

23. KAWAMOTO, H. Bunseki Kagaku, 17, 1139 (1968).

24. KAWAMOTO, H. and AKAIWA, H. Bunseki Kagaku, 18, 261 (1969).

25. MATH, K.S., BHATKI, K.S. and FREISER, H. Talanta, 16, 412 (1969).

26. AKAIWA, H., KAWAMOTO, H. and hiYAMUTA, E. Bull. Chem. Soc. Jpn. 54, 3409 (1981).

27. AKAIWA, H., KAWAmoto, $H$. and SUZUKI, K. Proceedings of the 43rd Annual Meeting of the Chemical Society of Japan, I , p. 382 (1981).

28. AKAIWA, H., KAWAMOTO, H. and YOSHIMATSU, E. Bull. Chem. Soc. Jpn., 52, 3718 (1979). 
29. KAWAMOTO, H. and AKAIWA, H. Unpublished Results.

30. IR VING, H.M.N.H. Ion Exchange and Solvent Extraction, Marcel Dekker, Inc., New York, p. 140 (1974).

31. AKAIWA, H., KAWAMOTO, H. and SAITOH, T. Nippon Kagaku Kaishi, 9I, 980 (1970).

32. AKAIWA, H., KAWAMOTO, H. and YOShIMATSU, E. Chem. Lett., 421 (1978).

33. AKAIWA, H. and KAWAMOTO, H. Anal. Chim. Acta, 48, 438 (1969).

34. AKAIWA, H., KAWAMOTO, H. and ISHII, T.J. Inorg. Nucl. Chem., 36, 2077 (1974).

35. AKAIWA, H. Chusyutsu Bunri Bunsekiho (Methods of Separation by Extraction), Kodansya, Tokyo, p. 105 (1972).

36. HEADRIDGE, J.B. and RICHARDSON, J. Analyst 94, 968 (1969).

37. BROVKO, I.A., NAZAROV, S.N. and RISH, M.A. Zhur. Anal. Khim., 29, 2387 (1974).

38. CRESSER, M.S. Solvent Extraction in Flame Spectroscopic Analysis, Butterworths, London, p. 51 (1978).

39. AKAIWA, H., KAWAMOTO, H., KOIZUMI, T. and SCHINDLER, P.W. Bunseki Kagaku, 31, E 151 (1982).

40. AKAIWA, H., KAWAMOTO, H. and HIYAMUTA, E. Bunseki Kagaku, 28, 477 (1979).

41. AKAIWA, H., KAWAMOTO, H. and KONISHI, M. Bunseki Kagaku, 28, 690 (1979).

42. MICHELL, J.W. and GANGES, R. Anal. Chem., 46, 503 (1974).

43. DE, A.K. and RAHAMAN, M.S. Anal. Chim. Acta, 27591 (1962).

44. KAWAMOTO, H., SAITOH, T. and AKAIWA, H. Proceedings of the 17th Annual Meeting of the Japan Society for Analytical Chemistry, p. B386 (1968).

45. KHOPKAR, S.M. and DE, A.K. Fresenius'Z. Anal. Chem., 171, 241 (1959).

46. AKAIWA, H., KAWAMOTO, H. and ABE, M. Bull. Chem. Soc. Jpn., 44, 117 (1971).

47. MARCUS, Y. and KERTES, A.S. Ion Exchange and Solvent Extraction of Metal Complexes, Wiley-Interscience, New York, p. 824 (1969). 